\title{
The quest for magnetic massive stars in the Magellanic Clouds
}

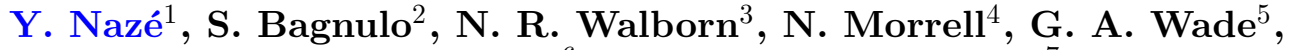 \\ M. K. Szymanski ${ }^{6}$ and R. H. D. Townsend ${ }^{7}$ \\ ${ }^{1}$ FNRS/Univ. Liège, Belgium; ${ }^{2}$ Armagh Obs., Ireland; ${ }^{3}$ STScI, USA $;{ }^{4}$ LCO, Chile $;{ }^{5}$ RMC, \\ Canada; ${ }^{6}$ Warsaw Univ., Poland; ${ }^{7}$ Univ. Wisconsin-Madison, USA
}

\begin{abstract}
The Of?p category was introduced more than 40 years ago to gather several Galactic stars with some odd properties. Since 2000, spectropolarimetry, high-resolution spectroscopy, long-term photometry, and X-ray observations have revealed their nature: magnetic oblique rotators - they all have magnetic fields that confine their winds. Several Of?p stars have now been detected in the Magellanic Clouds, likely the prototypes of magnetic massive stars at low metallicity. This contribution will present the most recent photometric, spectroscopic, and spectropolarimetric data, along with the first modeling of these objects.
\end{abstract}

Keywords. stars: early-type - stars: winds - stars: magnetic fields

Of?p are the sole class of (strongly) magnetic O-stars in the Galaxy. Five stars presenting the same spectral peculiarities were discovered in the Magellanic Clouds, and we investigated them in more detail to assess the impact of metallicity on the magnetism of massive stars. Photometric data from ASAS, EROS-2, and OGLE II-III-IV were analyzed using a modified Fourier algorithm, conditional entropy, binned analysis of variances and string length methods (Nazé et al. 2015). Spectroscopic data were obtained at LCO, AAT, ESO, and Siding Spr. Observ. (Walborn et al. 2015). Spectropolarimetric data were gathered with FORS2 and underwent several checks (Bagnulo et al., A\&A, in press): observation of standard magnetic stars and of non-magnetic field stars, use of null diagnostics. Individual results for each star are:

- $\mathrm{SMC159-2}(\mathrm{P}=14.91 \mathrm{~d})$ has very strong emissions (e.g. $\mathrm{EW}(\mathrm{H} \alpha) \sim 19 \AA)$, only rivalled by the most magnetic O-star NGC1624-2; $B_{z}>5 \mathrm{kG}$ is ruled out at maximum phase.

- 2 dFS936 ( $\mathrm{P}=1370 \mathrm{~d}+2 \mathrm{~d}$ because of line-of-sight object?) recently shows a large increase in emission strength and a small increase in brightness, an unexplained departure from an otherwise repetitive behaviour; $B_{z}<2.5 \mathrm{kG}$ at maximum emission phase.

- AV220 is varying (and currently declining) but no periodicity could be identified is the period long or are irregular variations possible for Of?p stars?; $B_{z}<3 \mathrm{kG}$.

- BI57 ( $\mathrm{P}=787 \mathrm{~d})$ shows a single maximum in optical line strength while having two photometric maxima, i.e. it is the first Of?p where photometric and spectroscopic behaviour are (partially) decoupled; $B_{z}<3 \mathrm{kG}$ (at a photometric max/spectroscopic min).

- LMC164-2 ( $\mathrm{P}=7.96 \mathrm{~d})$ has $B_{z}<1.7 \mathrm{kG}$ close to maximum phase.

Finally, we performed Monte-Carlo radiative transfer simulating light scattering in a circumstellar envelope following the confined wind structure simulated for HD191612, with initial photon emission launched from star at a random angle (Wade et al., in prep.). The derived lightcurves strongly depend on the inclination $i$ of the star's rotation axis and obliquity $\beta$ of the magnetic axis, helping to constrain the systems' geometries.

\section{References}

Nazé, Y., Walborn, N. R., Morrell, N., Wade, G. A. \& Szymanski, M. K. 2015, A\&6A, 577, A107 Walborn, N. R., Morrell, N. I., Nazé, Y., et al. 2015, AJ, 150, 99 